\title{
Reducing Sharp Fluctuations in Body Temperature and Optimizing Production Index of Broilers Using Dietary Electrolytes
}

\author{
Ibukun Olukorede Popoola ${ }^{1,2}$, Oluwabukola Rashidat Popoola ${ }^{1}$, Oluwaseyi Olamide Olajide ${ }^{1}$, \\ Akinyemi Alaba Adeyemi ${ }^{1}$, Queenesther Tolu Alegbejo ${ }^{1}$ \\ ${ }^{1}$ Department of Animal Science, University of Ibadan, Ibadan, Nigeria \\ ${ }^{2}$ Agricultural Research and Biometrics Department, Thisavrous Pyrgos Int. Resources, Oyo State, Nigeria \\ Email: *popoolaibukun@yahoo.com
}

How to cite this paper: Popoola, I.O., Popoola, O.R., Olajide, O.O., Adeyemi, A.A. and Alegbejo, Q.T. (2020) Reducing Sharp Fluctuations in Body Temperature and Optimizing Production Index of Broilers Using Dietary Electrolytes. Open Journal of Animal Sciences, 10, 266-277.

https://doi.org/10.4236/ojas.2020.102015

Received: January 25, 2020

Accepted: March 3, 2020

Published: March 6, 2020

Copyright $\odot 2020$ by author(s) and Scientific Research Publishing Inc. This work is licensed under the Creative Commons Attribution International License (CC BY 4.0).

http://creativecommons.org/licenses/by/4.0/

\begin{abstract}
A degree centigrade fall or rise in body temperature of broiler chickens is sufficient to reduce performance, alter sound physiological state and divert nutritional metabolism in quest for ensuring thermal balance. Thermoregulatory mechanisms of newly hatched chicks are poorly developed, and fluctuations in brooding temperature coupled with severe environmental temperatures in the tropics could threaten the survivability, production and economic integrity of fast-growing strains of broilers. However, information on the effect of sharp fluctuations in body temperature on nutrient intake and European production index (EPI) of broilers fed dietary electrolytes is scanty and thus investigated. Using a total of 300, one-day-old unsexed broiler chicks (Arbor Acre) that were randomly allotted to six dietary treatments (T1-210, T2-240, T3270, T4-300, T5-330 and T6-360 mEq/kg electrolyte balance), data on performance characteristics and their correlations with body temperature and EPI at prestarter, starter and finisher phases were determined using standard procedures. Data were analysed using descriptive statistics and ANOVA at $\alpha=0.05$. At prestarter phase, feed intake (FI) had a strong and positive relationship with protein intake $(\mathrm{r}=1.00 ; \mathrm{p}<0.01)$, EPI $(\mathrm{r}=0.96 ; \mathrm{p}<0.01)$ and potassium $(\mathrm{K})$ intake $(r=0.66 ; p<0.01)$, but was negatively correlated with temperature change $(r=-0.39 ; p<0.05)$. However, FI was not significantly correlated with body weight (BW), body weight gain (BWG), sodium $(\mathrm{Na})$ and water intake. At starter phase, EPI was positively correlated to $\mathrm{K}$ intake $(\mathrm{r}=0.38 ; \mathrm{p}$ $<0.05)$, but not $\mathrm{Na}$ and chloride $(\mathrm{Cl})$ intake. European production index of starter chicks could be enhanced by increasing the level of $\mathrm{K}$ intake through dietary supplementation up to $1.02 \%$. Weight gain was adversely affected by sharp changes in temperature at finisher phase. Increase in water intake may
\end{abstract}


not be a remedial tool in lowering sharp fluctuations in body temperature. However, sharp fluctuation in body temperature of broilers was reduced with DEB of 330, 270 and $240 \mathrm{mEq} / \mathrm{kg}$ at prestarter, starter and finisher phases, respectively.

\section{Keywords}

Temperature Change, Broilers, European Production Index, Dietary Electrolyte Balance, Heat Stress

\section{Introduction}

Rectal temperature has been a good indicator of body temperature of birds as it is the body temperature measured per rectum and it is accurate for measuring internal body temperature. De Rensis and Scaramuzzi (2003) [1] also noted that rectal temperature is an excellent indicator of the physiological state and is reliable in evaluating thermal balance of the body, however, homeostasis mechanisms can prevent a rise in rectal temperature. Perissinotto et al., (2007) [2] reported that body temperature is determined by the balance between heat loss and gain, and its value is obtained by measuring the rectal temperature. The thermoregulatory characteristics of poultry differ to some extent from those of mammals due to their high rate of metabolism and more intensive heat production relative to their lowered dissipating capacity of heat, as affected by feathers and absence of sweat glands. Evaporative cooling is therefore achieved exclusively by panting. Daghir (2009) [3] reported that after feathering, birds prefer mean ambient temperatures between $18^{\circ} \mathrm{C}$ to $22^{\circ} \mathrm{C}$ for growth and egg production, although the optimal temperature for feed efficiency is higher. The author noted further that the crucial temperature for poultry is $30^{\circ} \mathrm{C}$, because birds are still able to compensate for energy loss caused by lower feed intake through a relatively better feed conversion ratio and lowered basal metabolic rate. However, above $30^{\circ} \mathrm{C}$, nutrient intake declines such that birds are no longer able to compensate for it, and a showcase of rapid decline in production becomes vivid. Birds are homoeothermic like mammals, and they have to maintain a relatively constant temperature of their vital organs. Therefore, it becomes essential that heat be lost or conserved in response to changes in the environment. Athira et al. (2017) [4] noted that climate change has led to environmental stresses that affect efficient livestock production. A significant decrease in productive potential and an increase in physiological responses are observed in animals which are exposed to hot climates. According to Borges et al. (2003) [5], newly hatched chicks have body temperatures approximately $2.5^{\circ} \mathrm{C}$ below that of the adult bird, and it takes about a week post-hatch before the adult body temperature can be reached. This increase is related to feather cover and the increase in metabolic activities associated with growth. However, Borges et al. (2003) [5] stated that the use of dietary electrolytes could enhance broiler performance under heat stress, and noted 
further that regardless of ambient temperatures, increasing DEB in broiler rations stimulated feed intake, which may possibly be due to increasing $\mathrm{Na}^{+}$levels in diets $(0.15 \%$ to $0.45 \%)$. Mongin (1981) [6] reported that optimal chick growth performance, when fed purified diets, was achieved using DEB of around 250 $\mathrm{mEq} / \mathrm{kg}$, and an optimal electrolyte balance was found for feeds containing from 250 to $300 \mathrm{mEq} / \mathrm{kg}$. Murakami et al. (2001) [7] established with modern broiler strains and practical diets, an optimal DEB for starter phase to be between 246 and $315 \mathrm{mEq} / \mathrm{kg}$, showcasing the efficacy of dietary electrolyte balance for enhancing performance of broiler chickens. However, information on the effect of DEB on body temperature change and production index of fast growing strains of broiler chickens reared under severe heat stress condition is scanty. Therefore, this study was conducted to investigate the effect of sharp fluctuations in body temperature on nutrient intake and European production index (EPI) of broilers fed dietary electrolytes and also to determine the association between core performance parameters, body temperature and EPI of broilers under tropical conditions at prestarter, starter and finisher phases.

\section{Materials and Methods}

The study was carried out at the Teaching and Research Farm, University of Ibadan, Nigeria, after the experimental protocol was reviewed and approved by the Institutional Animal Care and Use Committee; and the Agricultural Biochemistry and Nutrition Unit of the Department of Animal Science. A total of 300, one-day old unsexed Arbor Acre broiler chicks were randomly allotted to six dietary treatments (mEq/kg: 210, 240, 270, 300, 330, 360 DEB) with five replicate groups in a Completely Randomized Design (CRD). Feed grade potassium chloride and sodium bicarbonate, and the inherent potassium, sodium and chloride ions in feed ingredients were the electrolyte sources computed for determining the aggregate DEB using the equations derived by Popoola and Iyayi (2018) [8]. The derived equations were based on assumptions opined by Popoola et al. (2020) [9] for an ideal DEB, affirming that not more than 30 to $140 \mathrm{mEq} / \mathrm{kg}$ DEB are required from mineral sources, with about 115 to $210 \mathrm{mEq} / \mathrm{kg}$ DEB obtainable from feed ingredients.

The derived equation of DEB is $\sum \mathrm{DEB}=\sum\left(\mathrm{Na}^{+}+\mathrm{K}^{+}\right)-\sum \mathrm{Cl}^{-} \cdots[\mathrm{y}][\mathrm{c}]$

where $[y]=$ mineral sources, and $[c]=$ other macro ions $(\mathrm{Ca}, \mathrm{Mg}, \mathrm{P}, \mathrm{S}$ etc) held constant.

$$
\sum \mathrm{DEB}=\imath \mathrm{DEB}+\varepsilon \mathrm{DEB}
$$

where $\Sigma \mathrm{DEB}=$ Aggregate $\mathrm{DEB} ; \iota \mathrm{DEB}=$ Inherent $\mathrm{DEB}$ in rations and $\varepsilon \mathrm{DEB}=\mathrm{DEB}$ in Electrolyte sources.

Feed intake was determined by giving a known quantity of feed to the birds and subtracting the left over for a given period from the quantity supplied. This difference was divided by the number of birds in a replicate group to estimate the feed intake per bird. Body weight gain of birds was determined by subtracting the initial weight for each week from the final weights with the aid of sensi- 
tive weighing scale. Drinking water was supplied to broilers as described by Popoola et al. (2019) [10]. European production index (EPI) was calculated using the formula

$$
\mathrm{EPI}=\frac{\text { Liveability }(\%) \times \text { Body weight }(\mathrm{kg})}{\text { Age }(\mathrm{d}) \times \mathrm{FCR}(\mathrm{kg} \text { feed } / \mathrm{kg} \text { gain })} \times 100
$$

Maximum and minimum average ambient temperature and relative humidity were monitored on a daily basis using a digital hygro-thermometer. Rectal temperature was measured in the morning (06:00-08:00 h) and afternoon (13:00-15:00 h), with the use of a digital rectal probe. Two birds per pen with body weight closest to the class mean weight were identified for body temperature measurement. Assay was conducted for sodium and potassium (Flame spectrophotometer), and chloride (titration) in diets fed to broiler chickens at different phases of growth (Lacroix et al., 1970) [11]. Data obtained were subjected to descriptive statistics and analysis of variance using SAS package (2012) [12]. Means for treatments in the analysis of variance were compared using Duncan Multiple range test and all statement of significance were based on probability level of 0.05 .

\section{Results}

The chemical composition of dietary treatments fed to broiler chickens at starter and finisher phases is shown in Table 1. Table 2 shows the growth performance, European production index and body temperature of broilers fed dietary electrolytes at prestarter phase. Feed intake (FI) of birds on $270 \mathrm{mEq} / \mathrm{kg}$ DEB was significantly $(\mathrm{p}<0.05)$ lower compared to $330 \mathrm{mEq} / \mathrm{kg}$ DEB. However, body weight (BW) and body weight gain (BWG) of birds were not affected by aggregate DEB treatments. Protein intake (PI) was observed to be lower in birds on $270 \mathrm{mEq} / \mathrm{kg}$ DEB. The European production index (EPI) observed in broilers on $270 \mathrm{mEq} / \mathrm{kg}$ (328.30) and $360 \mathrm{mEq} / \mathrm{kg}$ (338.86) were significantly $(\mathrm{p}<0.05)$ lower compared to $330 \mathrm{mEq} / \mathrm{kg}(369.39) \mathrm{DEB}$ at prestarter phase. Na intake was lowest $(\mathrm{p}<0.05)$ in birds on $210 \mathrm{mEq} / \mathrm{kg}(0.62 \mathrm{~g})$ and highest in those on $360 \mathrm{mEq} / \mathrm{kg}(2.20 \mathrm{~g})$ DEB. $\mathrm{K}$ intake in birds on $330 \mathrm{mEq} / \mathrm{kg}(4.70 \mathrm{~g})$ and $360 \mathrm{mEq} / \mathrm{kg}$ (4.62) was significantly $(\mathrm{p}<0.05)$ higher compared to other treatments. $\mathrm{Cl}$ intake observed in birds on $240 \mathrm{mEq} / \mathrm{kg}(1.22 \mathrm{~g})$ and $270 \mathrm{mEq} / \mathrm{kg}(1.35 \mathrm{~g})$ were similar and significantly ( $\mathrm{p}<0.05$ ) lower compared to $300(1.62 \mathrm{~g}), 330(1.98 \mathrm{~g})$, and $360 \mathrm{mEq} / \mathrm{kg}$ $(2.07 \mathrm{~g})$ DEB. Water intake (WI) values of birds on 210,240 , and $300 \mathrm{mEq} / \mathrm{kg}$ DEB were similar and significantly $(\mathrm{p}<0.05)$ lower compared to $360 \mathrm{mEq} / \mathrm{kg} \mathrm{DEB}$. The lowest $(\mathrm{p}<0.05)$ morning and afternoon rectal temperature $(\mathrm{RT})$ was observed in birds on $270 \mathrm{mEq} / \mathrm{kg}$. However, temperature change (TC) in broilers on 330 $\mathrm{mEq} / \mathrm{kg}\left(0.35^{\circ} \mathrm{C}\right) \mathrm{DEB}$ was significantly $(\mathrm{p}<0.05)$ lower compared to other treatments. At starter phase (Table 3 ), FI was not significantly ( $p>0.05$ ) affected by dietary treatments. However, BW was significantly higher in birds on $330 \mathrm{mEq} / \mathrm{kg}$ (679.68 g) DEB compared to other treatments except $270 \mathrm{mEq} / \mathrm{kg}$ (673.44 g). The PI, BWG, and EPI were not significantly ( $p>0.05)$ affected by DEB treatments 
Table 1. Chemical composition of diets fed to broilers at starter and finisher phases.

\begin{tabular}{|c|c|c|c|c|c|c|c|}
\hline Phase & Nutrients (\%) & $\mathrm{T} 1$ & $\mathrm{~T} 2$ & T3 & $\mathrm{T} 4$ & T5 & T6 \\
\hline \multirow{7}{*}{ Starter } & Crude protein & 22.90 & 22.95 & 23.10 & 22.93 & 22.79 & 22.89 \\
\hline & Crude fat & 5.29 & 5.26 & 5.44 & 5.54 & 5.46 & 5.66 \\
\hline & Calcium & 1.01 & 1.02 & 1.04 & 1.01 & 1.02 & 1.03 \\
\hline & Phosphorus & 0.82 & 0.83 & 0.89 & 0.81 & 0.83 & 0.82 \\
\hline & Sodium & 0.14 & 0.22 & 0.29 & 0.36 & 0.43 & 0.51 \\
\hline & Potassium & 0.82 & 0.87 & 0.92 & 0.97 & 1.02 & 1.07 \\
\hline & Chloride & 0.23 & 0.28 & 0.33 & 0.38 & 0.43 & 0.48 \\
\hline \multirow{7}{*}{ Finisher } & Crude protein & 21.78 & 21.67 & 21.84 & 21.79 & 21.66 & 21.85 \\
\hline & Crude fat & 4.24 & 4.20 & 4.15 & 4.11 & 4.21 & 4.09 \\
\hline & Calcium & 1.03 & 0.94 & 0.94 & 0.95 & 0.96 & 0.96 \\
\hline & Phosphorus & 0.78 & 0.77 & 0.78 & 0.78 & 0.78 & 0.78 \\
\hline & Sodium & 0.16 & 0.23 & 0.29 & 0.37 & 0.44 & 0.52 \\
\hline & Potassium & 0.83 & 0.85 & 0.89 & 0.95 & 0.99 & 1.04 \\
\hline & Chloride & 0.25 & 0.27 & 0.32 & 0.37 & 0.42 & 0.47 \\
\hline
\end{tabular}

T1 -210 mEq/kg, T2-240 mEq/kg, T3-270 mEq/kg, T4-300 mEq/kg, T5-330 mEq/kg, T6-360 mEq/kg, DEB-Dietary electrolyte balance.

Table 2. Growth performance, production index and body temperatures of heat-stressed broilers at prestarter phase.

\begin{tabular}{|c|c|c|c|c|c|c|c|c|}
\hline \multirow{2}{*}{ Parameters } & \multicolumn{8}{|c|}{ Dietary electrolyte balance (mEq/kg) } \\
\hline & 210 & 240 & 270 & 300 & 330 & 360 & SEM & p value \\
\hline FI (g/bird) & $443.16^{\mathrm{ab}}$ & $437.20^{\mathrm{ab}}$ & $409.28^{b}$ & $426.17^{\mathrm{ab}}$ & $460.82^{\mathrm{a}}$ & $431.66^{\mathrm{ab}}$ & 13.72 & 0.20 \\
\hline BW (g/bird) & 319.60 & 331.24 & 332.16 & 339.39 & 333.40 & 320.14 & 10.99 & 0.77 \\
\hline BWG (g/bird) & 283.40 & 294.66 & 295.80 & 303.17 & 297.10 & 284.54 & 10.96 & 0.78 \\
\hline PI (g/bird) & $101.93^{\mathrm{ab}}$ & $100.56^{\mathrm{ab}}$ & $94.13^{\mathrm{b}}$ & $98.02^{\mathrm{ab}}$ & $105.99^{\mathrm{a}}$ & $99.28^{\mathrm{ab}}$ & 3.16 & 0.20 \\
\hline EPI & $357.00^{\mathrm{ab}}$ & $351.04^{\mathrm{ab}}$ & $328.30^{\mathrm{b}}$ & $341.45^{\mathrm{ab}}$ & $369.39^{\mathrm{a}}$ & $338.86^{\mathrm{b}}$ & 9.44 & 0.06 \\
\hline $\mathrm{Na}$ intake (g/bird) & $0.62^{\mathrm{f}}$ & $0.96^{\mathrm{e}}$ & $1.19^{\mathrm{d}}$ & $1.53^{\mathrm{c}}$ & $1.98^{\mathrm{b}}$ & $2.20^{\mathrm{a}}$ & 0.05 & $<0.0001$ \\
\hline K intake (g/bird) & $3.63^{c}$ & $3.80^{\mathrm{bc}}$ & $3.77^{\mathrm{bc}}$ & $4.13^{\mathrm{b}}$ & $4.70^{\mathrm{a}}$ & $4.62^{\mathrm{a}}$ & 0.13 & $<0.0001$ \\
\hline $\mathrm{Cl}$ intake (g/bird) & $1.02^{\mathrm{d}}$ & $1.22^{\mathrm{c}}$ & $1.35^{\mathrm{c}}$ & $1.62^{\mathrm{b}}$ & $1.98^{\mathrm{a}}$ & $2.07^{\mathrm{a}}$ & 0.05 & $<0.0001$ \\
\hline $\begin{array}{l}\text { Water intake } \\
(\mathrm{mL} / \mathrm{bird} / \mathrm{d})\end{array}$ & $235.63^{c}$ & $261.93^{b}$ & $265.18^{b}$ & $265.69^{b}$ & $280.67^{\mathrm{ab}}$ & $297.16^{\mathrm{a}}$ & 7.67 & $<0.0001$ \\
\hline Morning RT $\left({ }^{\circ} \mathrm{C}\right)$ & $40.96^{\mathrm{a}}$ & $40.84^{\mathrm{a}}$ & $40.12^{\mathrm{c}}$ & $40.78^{\mathrm{a}}$ & $40.54^{\mathrm{b}}$ & $40.96^{\mathrm{a}}$ & 0.06 & $<0.0001$ \\
\hline Afternoon RT $\left({ }^{\circ} \mathrm{C}\right)$ & $41.41^{\mathrm{a}}$ & $41.45^{\mathrm{a}}$ & $40.69^{c}$ & $41.37^{\mathrm{a}}$ & $40.89^{\mathrm{b}}$ & $41.47^{\mathrm{a}}$ & 0.05 & $<0.0001$ \\
\hline $\mathrm{TC}\left({ }^{\circ} \mathrm{C}\right)$ & $0.46^{\mathrm{ab}}$ & $0.61^{\mathrm{a}}$ & $0.58^{\mathrm{a}}$ & $0.59^{\mathrm{a}}$ & $0.35^{\mathrm{b}}$ & $0.51^{\mathrm{ab}}$ & 0.06 & 0.03 \\
\hline
\end{tabular}

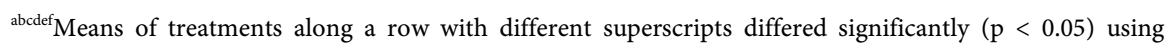
DMRT. FI-Feed intake, BW-Body weight, BWG-Body weight gain, PI-Protein intake, EPI-European production index, $\mathrm{Na}-$ Sodium, $\mathrm{K}$-Potassium, $\mathrm{Cl}$-Chloride, $\mathrm{RT}$-Rectal temperature, TC-Temperature change. 
Table 3. Growth performance, production index and body temperatures of heat-stressed broilers at starter phase.

\begin{tabular}{ccccccccc}
\hline \multirow{2}{*}{ Parameters } & \multicolumn{7}{c}{ Dietary electrolyte balance (mEq/kg) } \\
\cline { 2 - 9 } & 210 & 240 & 270 & 300 & 330 & 360 & SEM & p value \\
\hline FI (g/bird) & 424.00 & 420.70 & 441.00 & 445.89 & 419.00 & 423.00 & 3.97 & 0.53 \\
BW (g/bird) & $629.14^{\mathrm{bc}}$ & $659.80^{\mathrm{b}}$ & $673.44^{\mathrm{ab}}$ & $656.50^{\mathrm{b}}$ & $679.68^{\mathrm{a}}$ & $625.78^{\mathrm{c}}$ & 14.67 & 0.07 \\
BWG (g/bird) & 309.54 & 328.56 & 341.28 & 317.11 & 346.28 & 304.64 & 14.43 & 0.26 \\
PI (g/bird) & 97.52 & 96.76 & 101.43 & 102.55 & 96.37 & 97.29 & 2.87 & 0.53 \\
EPI & 411.90 & 403.24 & 414.87 & 448.38 & 391.68 & 416.17 & 19.74 & 0.48 \\
Na intake (g/bird) & $0.59^{\mathrm{f}}$ & $0.93^{\mathrm{e}}$ & $1.28^{\mathrm{d}}$ & $1.61^{\mathrm{c}}$ & $1.80^{\mathrm{b}}$ & $2.16^{\mathrm{a}}$ & 0.05 & $<0.0001$ \\
K intake (g/bird) & $3.48^{\mathrm{c}}$ & $3.66^{\mathrm{c}}$ & $4.06^{\mathrm{b}}$ & $4.33^{\mathrm{ab}}$ & $4.27^{\mathrm{ab}}$ & $4.53^{\mathrm{a}}$ & 0.12 & $<0.0001$ \\
Cl intake (g/bird) & $0.98^{\mathrm{e}}$ & $1.18^{\mathrm{d}}$ & $1.46^{\mathrm{c}}$ & $1.69^{\mathrm{b}}$ & $1.80^{\mathrm{b}}$ & $2.03^{\mathrm{a}}$ & 0.05 & $<0.0001$ \\
Water intake & $449.32^{\mathrm{b}}$ & $498.95^{\mathrm{a}}$ & $501.98^{\mathrm{a}}$ & $507.48^{\mathrm{a}}$ & $506.55^{\mathrm{a}}$ & $524.72^{\mathrm{a}}$ & 10.51 & $<0.0001$ \\
$(\mathrm{~mL} / \mathrm{bird} / \mathrm{d})$ & & & & & & & & \\
Morning RT $\left({ }^{\circ} \mathrm{C}\right)$ & $40.72^{\mathrm{a}}$ & $40.63^{\mathrm{a}}$ & $40.36^{\mathrm{b}}$ & $40.70^{\mathrm{a}}$ & $40.63^{\mathrm{a}}$ & $40.67^{\mathrm{a}}$ & 0.04 & $<0.0001$ \\
Afternoon RT $\left({ }^{\circ} \mathrm{C}\right)$ & $41.71^{\mathrm{a}}$ & $41.69^{\mathrm{a}}$ & $40.90^{\mathrm{c}}$ & $41.69^{\mathrm{a}}$ & $41.43^{\mathrm{b}}$ & $41.65^{\mathrm{a}}$ & 0.03 & $<0.0001$ \\
TC $\left({ }^{\circ} \mathrm{C}\right)$ & $0.99^{\mathrm{a}}$ & $1.05^{\mathrm{c}}$ & $0.54^{\mathrm{c}}$ & $0.99^{\mathrm{a}}$ & $0.79^{\mathrm{b}}$ & $0.98^{\mathrm{a}}$ & 0.04 & $<0.0001$ \\
\hline
\end{tabular}

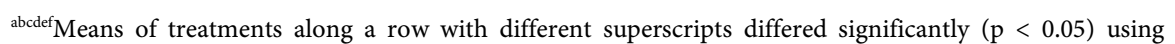
DMRT. FI-Feed intake, BW-Body weight, BWG-Body weight gain, PI-Protein intake, EPI-European production index, $\mathrm{Na}$-Sodium, $\mathrm{K}$-Potassium, $\mathrm{Cl}$-Chloride, $\mathrm{RT}$-Rectal temperature, $\mathrm{TC}-\mathrm{Temperature}$ change.

at starter phase. However, $\mathrm{Na}, \mathrm{K}$, and $\mathrm{Cl}$ intake were significantly $(\mathrm{p}<0.05)$ higher in birds on $360 \mathrm{mEq} / \mathrm{kg}$ DEB. Similar WI values were observed in birds on 240 (498.95), 270 (501.98), 300 (507.48), 330 (506.55) and $360 \mathrm{DEB}$ (524.72 mL/bird/d). However, birds on $270 \mathrm{mEq} / \mathrm{kg}$ DEB had the lowest $(\mathrm{p}<0.05) \mathrm{TC}$ of $0.54^{\circ} \mathrm{C}$.

At finisher phase (Table 4), FI was not significantly influenced by dietary treatments. However, BW observed in birds on $210 \mathrm{mEq} / \mathrm{kg}$ (1310.00) was significantly $(\mathrm{p}<0.05)$ lower compared to 240,270 , and $300 \mathrm{mEq} / \mathrm{kg}$ DEB. Similar values of BWG were observed in birds on 210 DEB (680.86 g) and 330 (676.72 g). The EPI was not significantly ( $p>0.05)$ affected by DEB treatments at finisher phase. However, $\mathrm{Na}, \mathrm{K}$, and $\mathrm{Cl}$ intake values were significantly $(\mathrm{p}<0.05)$ lower in birds on $210 \mathrm{mEq} / \mathrm{kg}$ DEB compared to other treatments. WI was lower $(\mathrm{p}<0.05)$ in 210 (1042.79), 240 (1068.70), and 300 (1052.86) compared to $360(1203.71 \mathrm{~mL})$. Morning and afternoon RT were highest $(\mathrm{p}<0.05)$ in birds on $210 \mathrm{mEq} / \mathrm{kg}$. However, $240 \mathrm{mEq} / \mathrm{kg}$ DEB resulted in significantly $(\mathrm{p}<0.05)$ lower TC $(0.89)$ compared to other treatments.

Table 5 shows the correlation between performance parameters and sharp fluctuations in body temperature of heat-stressed broilers at prestarter phase. Feed intake (FI) had a strong and positive relationship with protein intake $(\mathrm{r}=1.00 ; \mathrm{p}$ $<0.01)$, production index $(\mathrm{r}=0.96 ; \mathrm{p}<0.01)$ and potassium intake $(\mathrm{r}=0.66 ; \mathrm{p}<$ $0.01)$. However, FI was negatively correlated with temperature change $(r=-0.39$; $\mathrm{p}<0.05)$. It was observed that FI was not significantly $(\mathrm{p}>0.05)$ correlated with 
Table 4. Growth performance, production index and body temperatures of heat-stressed broilers at finisher phase.

\begin{tabular}{|c|c|c|c|c|c|c|c|c|}
\hline \multirow{2}{*}{ Parameters } & \multicolumn{8}{|c|}{ Dietary electrolyte balance $(\mathrm{mEq} / \mathrm{kg})$} \\
\hline & 210 & 240 & 270 & 300 & 330 & 360 & SEM & $\mathrm{p}$ value \\
\hline FI (g/bird) & 1531.80 & 1572.00 & 1543.00 & 1481.89 & 1528.00 & 1487.00 & 36.02 & 0.49 \\
\hline BW (g/bird) & $1310.00^{c}$ & $1485.12^{\mathrm{a}}$ & $1454.16^{\mathrm{ab}}$ & $1382.75^{\mathrm{b}}$ & $1356.40^{\mathrm{bc}}$ & $1344.91^{\mathrm{bc}}$ & 39.27 & 0.03 \\
\hline BWG (g/bird) & $680.86^{\mathrm{b}}$ & $825.32^{\mathrm{a}}$ & $780.72^{\mathrm{ab}}$ & $726.26^{\mathrm{ab}}$ & $676.72^{\mathrm{b}}$ & $719.13^{\mathrm{ab}}$ & 35.82 & 0.04 \\
\hline PI (g/bird) & 337.00 & 345.84 & 339.46 & 326.02 & 336.16 & 327.14 & 7.93 & 0.49 \\
\hline EPI & 849.82 & 812.81 & 822.05 & 809.29 & 875.85 & 798.05 & 27.82 & 0.38 \\
\hline $\mathrm{Na}$ intake (g/bird) & $2.45^{\mathrm{f}}$ & $3.62^{\mathrm{e}}$ & $4.47^{\mathrm{d}}$ & $5.48^{\mathrm{c}}$ & $6.72^{\mathrm{b}}$ & $7.73^{\mathrm{a}}$ & 0.14 & $<0.0001$ \\
\hline $\mathrm{K}$ intake (g/bird) & $12.71^{\mathrm{c}}$ & $13.36^{\mathrm{bc}}$ & $13.73^{\mathrm{bc}}$ & $14.08^{\mathrm{b}}$ & $15.13^{\mathrm{a}}$ & $15.46^{\mathrm{a}}$ & 0.34 & $<0.0001$ \\
\hline $\mathrm{Cl}$ intake (g/bird) & $3.83^{\mathrm{f}}$ & $4.24^{\mathrm{e}}$ & $4.94^{\mathrm{d}}$ & $5.48^{\mathrm{c}}$ & $6.42^{\mathrm{b}}$ & $6.99^{\mathrm{a}}$ & 0.13 & $<0.0001$ \\
\hline Water intake $(\mathrm{mL} / \mathrm{bird} / \mathrm{d})$ & $1042.79^{\mathrm{b}}$ & $1068.70^{\mathrm{b}}$ & $1103.73^{\mathrm{ab}}$ & $1052.86^{\mathrm{b}}$ & $1133.11^{\mathrm{ab}}$ & $1203.71^{\mathrm{a}}$ & 33.04 & 0.02 \\
\hline Morning RT $\left({ }^{\circ} \mathrm{C}\right)$ & $41.01^{\mathrm{a}}$ & $40.48^{\mathrm{d}}$ & $40.74^{\mathrm{c}}$ & $40.83^{\mathrm{b}}$ & $40.94^{\mathrm{a}}$ & $40.85^{\mathrm{b}}$ & 0.03 & $<0.0001$ \\
\hline Afternoon $\mathrm{RT}\left({ }^{\circ} \mathrm{C}\right)$ & $42.02^{\mathrm{a}}$ & $41.36^{\mathrm{d}}$ & $41.72^{\mathrm{c}}$ & $41.87^{\mathrm{b}}$ & $41.91^{\mathrm{b}}$ & $41.89^{\mathrm{b}}$ & 0.03 & $<0.0001$ \\
\hline $\mathrm{TC}\left({ }^{\circ} \mathrm{C}\right)$ & $1.01^{\mathrm{a}}$ & $0.89^{\mathrm{b}}$ & $0.98^{\mathrm{ab}}$ & $1.04^{\mathrm{a}}$ & $0.96^{\mathrm{ab}}$ & $1.05^{\mathrm{a}}$ & 0.04 & 0.07 \\
\hline
\end{tabular}

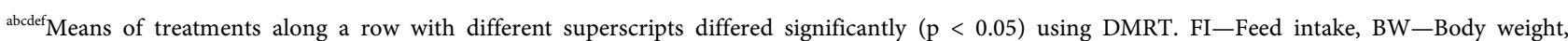
BWG-Body weight gain, PI-Protein intake, EPI-European production index, Na-Sodium, K-Potassium, Cl—Chloride, RT-Rectal temperature, TC-Temperature change.

Table 5. Correlation between performance parameters and sharp flunctuations in body temperature of heat-stressed broilers at prestarter phase.

\begin{tabular}{|c|c|c|c|c|c|c|c|c|c|c|c|c|}
\hline Parameters & FI & BW & BWG & PI & $\begin{array}{l}\text { Prod. } \\
\text { index }\end{array}$ & $\begin{array}{c}\mathrm{Na} \\
\text { intake }\end{array}$ & $\begin{array}{c}\mathrm{K} \\
\text { intake }\end{array}$ & $\begin{array}{c}\mathrm{Cl} \\
\text { intake }\end{array}$ & $\begin{array}{l}\text { Water } \\
\text { intake }\end{array}$ & $\begin{array}{c}\text { Morning } \\
\text { RT }\end{array}$ & $\begin{array}{c}\text { Afternoon } \\
\text { RT }\end{array}$ & TC \\
\hline FI & & $0.29^{\mathrm{ns}}$ & $0.29^{\mathrm{ns}}$ & $1.00^{* *}$ & $0.96^{* *}$ & $0.25^{\mathrm{ns}}$ & $0.66^{* *}$ & $0.35^{\text {ns }}$ & $0.01^{\mathrm{ns}}$ & $0.20^{\mathrm{ns}}$ & $0.08^{\text {ns }}$ & $-0.39^{*}$ \\
\hline BW & $0.29^{\mathrm{ns}}$ & & $0.99^{* *}$ & $0.29^{\text {ns }}$ & $0.23^{\mathrm{ns}}$ & $0.10^{\text {ns }}$ & $0.22^{\mathrm{ns}}$ & $0.13^{\text {ns }}$ & 0.02 & $-0.15^{\mathrm{ns}}$ & $-0.12^{\text {ns }}$ & $0.09^{\text {ns }}$ \\
\hline BWG & $0.29^{\mathrm{ns}}$ & $0.99^{* *}$ & & $0.29^{\mathrm{ns}}$ & $0.22^{\mathrm{ns}}$ & $0.11^{\mathrm{ns}}$ & $0.22^{\mathrm{ns}}$ & $0.14^{\mathrm{ns}}$ & $0.03^{\mathrm{ns}}$ & $-0.15^{\mathrm{ns}}$ & $-0.12^{\mathrm{ns}}$ & $0.09^{\mathrm{ns}}$ \\
\hline PI & $1.00^{* *}$ & $0.29^{\mathrm{ns}}$ & $0.29^{\mathrm{ns}}$ & & $0.96^{\star *}$ & $0.25^{\mathrm{ns}}$ & $0.66^{\star *}$ & $0.35^{\mathrm{ns}}$ & $0.01^{\mathrm{ns}}$ & $0.20^{\mathrm{ns}}$ & $0.08^{\text {ns }}$ & $-0.39^{*}$ \\
\hline Prod. index & $0.96^{* *}$ & $0.23^{\mathrm{ns}}$ & $0.22^{\mathrm{ns}}$ & $0.96^{* *}$ & & $0.19^{\text {ns }}$ & $0.59^{\star *}$ & $0.29^{\text {ns }}$ & $-0.39^{\text {ns }}$ & $0.25^{\mathrm{ns}}$ & $0.15^{\text {ns }}$ & $-0.31^{\mathrm{ns}}$ \\
\hline $\mathrm{Na}$ intake & $0.25^{\mathrm{ns}}$ & $0.10^{\mathrm{ns}}$ & $0.11^{\mathrm{ns}}$ & $0.25^{\mathrm{ns}}$ & $0.19^{\mathrm{ns}}$ & & $0.89^{\star *}$ & $0.99^{* *}$ & $0.93^{* *}$ & $-0.02^{\mathrm{ns}}$ & $-0.10^{\mathrm{ns}}$ & $-0.30^{\mathrm{ns}}$ \\
\hline $\mathrm{K}$ intake & $0.66^{\star *}$ & $0.22^{\mathrm{ns}}$ & $0.22^{\mathrm{ns}}$ & $0.66^{* *}$ & $0.59^{* *}$ & $0.89^{* *}$ & & $0.94^{\star *}$ & $0.72^{\star \star}$ & $0.08^{\mathrm{ns}}$ & $-0.05^{\mathrm{ns}}$ & $-0.43^{\star}$ \\
\hline $\mathrm{Cl}$ intake & $0.35^{\mathrm{ns}}$ & $0.13^{\text {ns }}$ & $0.14^{\mathrm{ns}}$ & $0.35^{\mathrm{ns}}$ & $0.29^{\mathrm{ns}}$ & $0.99^{* *}$ & $0.94^{* *}$ & & $0.89^{* *}$ & $0.01^{\mathrm{ns}}$ & $-0.09^{\text {ns }}$ & $-0.35^{\mathrm{ns}}$ \\
\hline Water intake & $0.01^{\mathrm{ns}}$ & $0.02^{\mathrm{ns}}$ & $0.03^{\text {ns }}$ & $0.01^{\mathrm{ns}}$ & $-0.04^{\mathrm{ns}}$ & $0.93^{* *}$ & $0.72^{\star *}$ & $0.89^{* *}$ & & $-0.07^{\text {ns }}$ & $-0.09^{\text {ns }}$ & $-0.10^{\mathrm{ns}}$ \\
\hline Morning RT & $0.20^{\mathrm{ns}}$ & $-0.15^{\text {ns }}$ & $-0.15^{\mathrm{ns}}$ & $0.20^{\mathrm{ns}}$ & $0.25^{\mathrm{ns}}$ & $-0.02^{\mathrm{ns}}$ & $0.08^{\text {ns }}$ & $0.01^{\mathrm{ns}}$ & $-0.07^{\mathrm{ns}}$ & & $0.96^{* *}$ & $-0.03^{\text {ns }}$ \\
\hline Afternoon RT & $0.08^{\mathrm{ns}}$ & $-0.12^{\text {ns }}$ & $-0.12^{\mathrm{ns}}$ & $0.08^{\mathrm{ns}}$ & $0.15^{\mathrm{ns}}$ & $-0.10^{\text {ns }}$ & $-0.05^{\mathrm{ns}}$ & $-0.09^{\text {ns }}$ & $-0.09^{\text {ns }}$ & $0.96^{* *}$ & & $0.27^{\mathrm{ns}}$ \\
\hline TC & $-0.39^{\star}$ & $0.09^{\text {ns }}$ & $0.09^{\text {ns }}$ & $-0.39^{\star}$ & $-0.31^{\text {ns }}$ & $-0.30^{\text {ns }}$ & $-0.43^{*}$ & $-0.35^{\mathrm{ns}}$ & $-0.10^{\text {ns }}$ & $-0.03^{\text {ns }}$ & $0.27^{\mathrm{ns}}$ & \\
\hline
\end{tabular}

${ }^{* *} \mathrm{p}<0.01,{ }^{*} \mathrm{p}<0.05, \mathrm{~ns}=$ not significant. FI-feed intake, BW—body weight, BWG-body weight gain, PI—Protein intake, Prod.-production, $\mathrm{Na}$-sodium, $\mathrm{K}$-potassium, $\mathrm{Cl}$-chloride, $\mathrm{RT}$-rectal temperature, $\mathrm{TC}$-temperature change.

BW, BWG, Na intake, water intake, morning and afternoon RT. The BW observed in broilers at prestarter phase was not significantly $(\mathrm{p}>0.05)$ correlated with $\mathrm{Na}$, $\mathrm{K}$, and $\mathrm{Cl}$ intake. Protein intake had a strong and positive relationship with pro- 
duction index $(\mathrm{r}=0.96 ; \mathrm{p}<0.01)$ and $\mathrm{K}$ intake $(\mathrm{r}=0.66 ; \mathrm{p}<0.01)$, but was negatively correlated to TC $(r=-0.39 ; \mathrm{p}<0.05)$. The $\mathrm{Na}(\mathrm{r}=0.93 ; \mathrm{p}<0.01), \mathrm{K}(\mathrm{r}=$ $0.72 ; \mathrm{p}<0.01)$, and $\mathrm{Cl}(\mathrm{r}=0.89 ; \mathrm{p}<0.01)$ had a strong and positive relationship with water intake of heat-stressed birds at prestarter phase. Temperature change was negatively correlated to potassium intake $(r=-0.43 ; p<0.05)$.

Table 6 shows the correlation between performance parameters and sharp fluctuations in body temperature of heat-stressed broilers at starter phase. The FI was positively correlated with BW $(\mathrm{r}=0.52 ; \mathrm{p}<0.01)$, protein intake $(\mathrm{r}=1.00 ; \mathrm{p}$ $<0.01)$, production index $(\mathrm{r}=0.59 ; \mathrm{p}<0.01)$ and $\mathrm{K}$ intake $(\mathrm{r}=0.58 ; \mathrm{p}<0.01)$. The BW was not significantly ( $\mathrm{p}>0.05$ ) correlated to morning or afternoon rectal temperature and temperature change. Production index was positively correlated to $\mathrm{K}$ intake $(\mathrm{r}=0.38 ; \mathrm{p}<0.05)$, but not $\mathrm{Na}$ and $\mathrm{Cl}$ intake. Water intake had a strong and positive relationship with $\mathrm{Na}(\mathrm{r}=0.85 ; \mathrm{p}<0.01), \mathrm{K}(\mathrm{r}=0.72 ; \mathrm{p}<$ $0.01)$ and $\mathrm{Cl}(\mathrm{r}=0.83 ; \mathrm{p}<0.01)$ intake at starter phase. Table 7 shows the correlation between performance parameters and sharp fluctuations in body temperature of heat-stressed broilers at finisher phase. Feed intake was negatively correlated with temperature change $(r=-0.36 ; p<0.05)$, but had a strong and positive relationship with production index $(\mathrm{r}=0.60 ; \mathrm{p}<0.01)$. The $\mathrm{BW}$ was negatively correlated to morning temperature $(r=-0.57 ; p<0.01)$, afternoon temperature $(\mathrm{r}=-0.57 ; \mathrm{p}<0.01)$ and temperature change $(\mathrm{r}=-0.45, \mathrm{p}<0.05)$. A strong, but negative correlation existed between body weight gain and temperature change $(\mathrm{r}=-0.39 ; \mathrm{p}<0.05)$ in heat-stressed birds at finisher phase. Sodium intake had a strong and positive relationship with temperature change $(r=0.39 ; \mathrm{p}<0.05)$.

Table 6. Correlation between performance parameters and sharp flunctuations in body temperature of heat-stressed broilers at starter phase.

\begin{tabular}{|c|c|c|c|c|c|c|c|c|c|c|c|c|}
\hline Parameters & FI & BW & BWG & PI & $\begin{array}{l}\text { Prod. } \\
\text { index }\end{array}$ & $\begin{array}{c}\mathrm{Na} \\
\text { intake }\end{array}$ & $\begin{array}{c}\mathrm{K} \\
\text { intake }\end{array}$ & $\begin{array}{c}\mathrm{Cl} \\
\text { intake }\end{array}$ & $\begin{array}{l}\text { Water } \\
\text { intake }\end{array}$ & $\begin{array}{c}\text { Morning } \\
\text { RT }\end{array}$ & $\begin{array}{c}\text { Afternoon } \\
\text { RT }\end{array}$ & $\mathrm{TC}$ \\
\hline FI & & $0.52^{\star \star}$ & $0.31^{\mathrm{ns}}$ & $1.00^{\star *}$ & $0.59^{\star *}$ & $0.17^{\mathrm{ns}}$ & $0.58^{\star \star}$ & $0.26^{\mathrm{ns}}$ & $0.05^{\mathrm{ns}}$ & $-0.15^{\text {ns }}$ & $-0.15^{\mathrm{ns}}$ & $-0.14^{\mathrm{ns}}$ \\
\hline BW & $0.52^{* *}$ & & $0.78^{* *}$ & $0.52^{* *}$ & $0.09^{\text {ns }}$ & $0.12^{\mathrm{ns}}$ & $0.33^{\mathrm{ns}}$ & $0.17^{\mathrm{ns}}$ & $-0.16^{\mathrm{ns}}$ & $-0.32^{\mathrm{ns}}$ & $-0.34^{\mathrm{ns}}$ & $-0.34^{\mathrm{ns}}$ \\
\hline BWG & $0.31^{\mathrm{ns}}$ & $0.78^{\star *}$ & & $0.31^{\mathrm{ns}}$ & $-0.47^{\star *}$ & $0.04^{\mathrm{ns}}$ & $0.17^{\mathrm{ns}}$ & $0.07^{\mathrm{ns}}$ & $0.08^{\mathrm{ns}}$ & $-0.29^{\mathrm{ns}}$ & $-0.32^{\mathrm{ns}}$ & $-0.32^{\mathrm{ns}}$ \\
\hline PI & $1.00^{* *}$ & $0.52^{\star \star}$ & 0.31 & & $0.59^{\star *}$ & $0.17^{\text {ns }}$ & $0.58^{\mathrm{ns}}$ & $0.26^{\mathrm{ns}}$ & $0.05^{\mathrm{ns}}$ & $-0.15^{\mathrm{ns}}$ & $-0.15^{\mathrm{ns}}$ & $-0.14^{\mathrm{ns}}$ \\
\hline Prod. index & $0.59^{* *}$ & $0.09^{\mathrm{ns}}$ & $-0.47^{\star *}$ & $0.59^{* *}$ & & $0.14^{\mathrm{ns}}$ & $0.38^{*}$ & $0.19^{\text {ns }}$ & $0.05^{\mathrm{ns}}$ & $0.06^{\mathrm{ns}}$ & $0.07^{\mathrm{ns}}$ & $0.07^{\mathrm{ns}}$ \\
\hline Na intake & $0.17^{\mathrm{ns}}$ & $0.12^{\mathrm{ns}}$ & $0.04^{\mathrm{ns}}$ & $0.17^{\mathrm{ns}}$ & $0.14^{\mathrm{ns}}$ & & $0.90^{\star *}$ & $0.99^{* *}$ & $0.85^{\star \star}$ & $0.01^{\mathrm{ns}}$ & $-0.07^{\mathrm{ns}}$ & $-0.12^{\mathrm{ns}}$ \\
\hline $\mathrm{K}$ intake & $0.58^{\star *}$ & $0.33^{\text {ns }}$ & $0.17^{\mathrm{ns}}$ & $0.58^{\star *}$ & $0.38^{* *}$ & $0.90^{* *}$ & & $0.94^{\star *}$ & $0.72^{\star \star}$ & $-0.05^{\text {ns }}$ & $-0.12^{\mathrm{ns}}$ & $-0.16^{\mathrm{ns}}$ \\
\hline $\mathrm{Cl}$ intake & $0.26^{\mathrm{ns}}$ & $0.17^{\mathrm{ns}}$ & $0.07^{\mathrm{ns}}$ & $0.26^{\mathrm{ns}}$ & $0.19^{\text {ns }}$ & $0.99^{* *}$ & $0.94^{\star *}$ & & $0.83^{\star *}$ & $0.003^{\mathrm{ns}}$ & $-0.08^{\mathrm{ns}}$ & $-0.13^{\mathrm{ns}}$ \\
\hline Water intake & $0.05^{\text {ns }}$ & $0.16^{\mathrm{ns}}$ & $0.08^{\mathrm{ns}}$ & $0.05^{\mathrm{ns}}$ & $0.05^{\mathrm{ns}}$ & $0.85^{\star *}$ & $0.72^{\star \star}$ & $0.83^{* *}$ & & $-0.22^{\mathrm{ns}}$ & $-0.18^{\mathrm{ns}}$ & $-0.14^{\mathrm{ns}}$ \\
\hline Morning RT & $-0.15^{\text {ns }}$ & $-0.32^{\mathrm{ns}}$ & $-0.29^{\text {ns }}$ & $-0.15^{\mathrm{ns}}$ & $0.06^{\mathrm{ns}}$ & $0.01^{\mathrm{ns}}$ & $-0.05^{\mathrm{ns}}$ & $0.003^{\text {ns }}$ & $-0.22^{\mathrm{ns}}$ & & $0.96^{* *}$ & $0.89^{* *}$ \\
\hline Afternoon RT & $-0.15^{\mathrm{ns}}$ & $-0.34^{\mathrm{ns}}$ & $-0.32^{\mathrm{ns}}$ & $-0.15^{\mathrm{ns}}$ & $0.07^{\mathrm{ns}}$ & $-0.07^{\mathrm{ns}}$ & $-0.12^{\mathrm{ns}}$ & $-0.08^{\mathrm{ns}}$ & $-0.18^{\mathrm{ns}}$ & $0.96^{\star *}$ & & $0.98^{\star *}$ \\
\hline TC & $-0.14^{\mathrm{ns}}$ & $-0.34^{\mathrm{ns}}$ & $-0.32^{\mathrm{ns}}$ & $-0.14^{\mathrm{ns}}$ & $0.07^{\mathrm{ns}}$ & $-0.12^{\mathrm{ns}}$ & $-0.16^{\mathrm{ns}}$ & $-0.13^{\text {ns }}$ & $-0.14^{\mathrm{ns}}$ & $0.89^{* *}$ & $0.98^{* *}$ & \\
\hline
\end{tabular}

${ }^{* *} \mathrm{p}<0.01,{ }^{*} \mathrm{p}<0.05, \mathrm{~ns}=$ not significant. FI-feed intake, BW-body weight, BWG-body weight gain, PI-Protein intake, Prod.-production, $\mathrm{Na}$-sodium, $\mathrm{K}$ - potassium, $\mathrm{Cl}$-chloride, $\mathrm{RT}$-rectal temperature, $\mathrm{TC}$-temperature change. 
Table 7. Correlation between performance parameters and sharp flunctuations in body temperature of heat-stressed broilers at finisher phase.

\begin{tabular}{|c|c|c|c|c|c|c|c|c|c|c|c|c|}
\hline Parameters & FI & BW & BWG & PI & $\begin{array}{l}\text { Prod. } \\
\text { index }\end{array}$ & $\begin{array}{c}\mathrm{Na} \\
\text { intake }\end{array}$ & $\begin{array}{c}\mathrm{K} \\
\text { intake }\end{array}$ & $\begin{array}{c}\mathrm{Cl} \\
\text { intake }\end{array}$ & $\begin{array}{l}\text { Water } \\
\text { intake }\end{array}$ & $\begin{array}{c}\text { Morning } \\
\text { RT }\end{array}$ & $\begin{array}{c}\text { Afternoon } \\
\text { RT }\end{array}$ & TC \\
\hline FI & & $0.45^{\star}$ & $0.33^{\text {ns }}$ & $1.00^{* *}$ & $0.60^{* *}$ & $-0.12^{\mathrm{ns}}$ & $0.35^{\mathrm{ns}}$ & $-0.04^{\mathrm{ns}}$ & $-0.14^{\mathrm{ns}}$ & $-0.22^{\mathrm{ns}}$ & -0.27 & $-0.36^{\star}$ \\
\hline BW & $0.45^{*}$ & & $0.94^{* *}$ & $0.45^{\star}$ & $-0.24^{\text {ns }}$ & $-0.09^{\text {ns }}$ & $0.08^{\text {ns }}$ & $-0.10^{\text {ns }}$ & $-0.11^{\mathrm{ns}}$ & $-0.57^{\star *}$ & $-0.57^{\star *}$ & $-0.45^{\star}$ \\
\hline BWG & $0.33^{\text {ns }}$ & $0.94^{\star *}$ & & $0.33^{\text {ns }}$ & $-0.49^{* *}$ & $-0.13^{\mathrm{ns}}$ & $-0.01^{\mathrm{ns}}$ & $-0.15^{\mathrm{ns}}$ & $-0.09^{\text {ns }}$ & $-0.58^{\star *}$ & $-0.56^{\star *}$ & $-0.39^{\star}$ \\
\hline PI & $1.00^{* *}$ & $0.45^{\star}$ & $0.33^{\text {ns }}$ & & $0.60^{* *}$ & $-0.12^{\mathrm{ns}}$ & $0.35^{\mathrm{ns}}$ & $-0.04^{\mathrm{ns}}$ & $-0.14^{\mathrm{ns}}$ & $-0.22^{\mathrm{ns}}$ & $-0.27^{\mathrm{ns}}$ & $-0.36^{\star}$ \\
\hline Prod. index & $0.60^{* *}$ & $-0.24^{\mathrm{ns}}$ & $-0.49^{* *}$ & $0.60^{* *}$ & & $0.02^{\mathrm{ns}}$ & $0.32^{\mathrm{ns}}$ & $0.09^{\mathrm{ns}}$ & $-0.07^{\mathrm{ns}}$ & $0.23^{\mathrm{ns}}$ & $0.17^{\mathrm{ns}}$ & $-0.06^{\mathrm{ns}}$ \\
\hline $\mathrm{Na}$ intake & $-0.12^{\text {ns }}$ & $-0.09^{\mathrm{ns}}$ & $-0.13^{\text {ns }}$ & $-0.12^{\mathrm{ns}}$ & $0.02^{\mathrm{ns}}$ & & $0.89^{* *}$ & $0.99^{* *}$ & $0.84^{\star *}$ & $0.18^{\mathrm{ns}}$ & $0.24^{\mathrm{ns}}$ & $0.39^{*}$ \\
\hline $\mathrm{K}$ intake & $0.35^{\mathrm{ns}}$ & $0.08^{\mathrm{ns}}$ & $-0.01^{\mathrm{ns}}$ & $0.35^{\mathrm{ns}}$ & $0.32^{\mathrm{ns}}$ & $0.89^{* *}$ & & $0.92^{\star *}$ & $0.71^{\star *}$ & $0.15^{\mathrm{ns}}$ & $0.19^{\text {ns }}$ & $0.27^{\mathrm{ns}}$ \\
\hline $\mathrm{Cl}$ intake & $-0.04^{\mathrm{ns}}$ & $-0.10^{\mathrm{ns}}$ & $-0.15^{\mathrm{ns}}$ & $-0.04^{\mathrm{ns}}$ & $0.09^{\text {ns }}$ & $0.99^{* *}$ & $0.92^{* *}$ & & $0.84^{\star *}$ & $0.25^{\mathrm{ns}}$ & $0.31^{\mathrm{ns}}$ & $0.43^{*}$ \\
\hline Water intake & $-0.14^{\mathrm{ns}}$ & $-0.11^{\mathrm{ns}}$ & $-0.09^{\text {ns }}$ & $-0.14^{\mathrm{ns}}$ & $-0.07^{\mathrm{ns}}$ & $0.84^{* *}$ & $0.71^{* *}$ & $0.84^{* *}$ & & $0.11^{\mathrm{ns}}$ & $0.15^{\mathrm{ns}}$ & $0.25^{\mathrm{ns}}$ \\
\hline Morning RT & $-0.22^{\mathrm{ns}}$ & $-0.57^{\star *}$ & $-0.58^{\star *}$ & $-0.22^{\mathrm{ns}}$ & $0.23^{\mathrm{ns}}$ & $0.18^{\text {ns }}$ & $0.15^{\mathrm{ns}}$ & $0.25^{\mathrm{ns}}$ & $0.11^{\mathrm{ns}}$ & & $0.99^{* *}$ & $0.74^{\star *}$ \\
\hline Afternoon RT & $-0.27^{\mathrm{ns}}$ & $-0.57^{\star *}$ & $-0.56^{\star *}$ & $-0.27^{\mathrm{ns}}$ & $0.17^{\mathrm{ns}}$ & $0.24^{\mathrm{ns}}$ & $0.19^{\text {ns }}$ & $0.31^{\mathrm{ns}}$ & $0.15^{\mathrm{ns}}$ & $0.99^{* *}$ & & $0.85^{\star *}$ \\
\hline TC & $-0.36^{*}$ & $-0.45^{*}$ & $-0.39^{\star}$ & $-0.36^{\mathrm{ns}}$ & $-0.06^{\mathrm{ns}}$ & $0.39^{*}$ & $0.27^{\mathrm{ns}}$ & $0.43^{*}$ & $0.25^{\mathrm{ns}}$ & $0.74^{* *}$ & $0.85^{* *}$ & \\
\hline
\end{tabular}

${ }^{* *} \mathrm{p}<0.01,{ }^{*} \mathrm{p}<0.05, \mathrm{~ns}=$ not significant. FI-feed intake, BW-body weight, BWG-body weight gain, PI-Protein intake, Prod.-production, $\mathrm{Na}$-sodium, $\mathrm{K}$-potassium, $\mathrm{Cl}$-chloride, $\mathrm{RT}$-rectal temperature, $\mathrm{TC}$-temperature change.

Also, $\mathrm{Cl}$ intake was positively correlated to temperature change $(\mathrm{r}=0.43 ; \mathrm{p}<$ $0.01)$ at finisher phase. However, $\mathrm{K}$ intake had no significant $(\mathrm{p}>0.05)$ relationship with temperature change at finisher phase. Water intake was not significantly $(p>0.05)$ correlated to changes in body temperature in heat-stressed broilers at finisher phase, but had a strong relationship with intake of $\mathrm{Na}(\mathrm{r}=0.84 ; \mathrm{p}<$ $0.01), \mathrm{K}(\mathrm{r}=0.71 ; \mathrm{p}<0.01)$ and $\mathrm{Cl}(\mathrm{r}=0.84 ; \mathrm{p}<0.01)$.

\section{Discussion}

The results obtained from current study were consistent with the report of Yalçin et al. (2001) [13] who observed that birds reared under high ambient temperatures tend to reduce feed consumption and increase water consumption; and this behavioral mechanism have been found to be more prominent in birds with higher body weight and growth rate (Deep and Cahaner, 2001) [14]. Thermoregulation is the ability of an animal to maintain body temperature in cold or hot environments, without affecting energy metabolism. Under cold climate, the body burns more nutrients and increase the rate of heat production to compensate for higher heat loss caused by the lower ambient temperatures. The results of present study agree with the observations of Plavnik and Yahav (1998) [15] who noted that for younger chicks with poorly developed thermoregulatory mechanism, higher ambient temperatures in the tropics as well as higher DEB can be beneficial to the survival of chicks at an early stage of growth. However, Temperatures comfortable for broiler chicks at early stage of life ranges from $33^{\circ} \mathrm{C}$ to $35^{\circ} \mathrm{C}$, and temperatures higher than these may induce a condition known as hyperthermia, coupled with dehydration, lower feed consumption and retarded growth. On the 
other hand, lower environmental temperatures induce hypothermia and often result in pulmonary hypertension in broilers. Sejian et al. (2012) [16] reported that environmental factors such as temperature, relative humidity and solar radiation has both direct and indirect effects on livestock production. The results of present study corroborate the assertions of Borges et al. (2003) [5], who noted that increase in body temperature of broiler chickens at finisher phase is related to feather cover and an increase in metabolic activities associated with growth, compared to prestarter chicks with body temperatures approximately $2.5^{\circ} \mathrm{C}$ lower than that of the adult bird. The results of current study indicated EPI values that showcased uniformity in body weight gain and healthy state of flock, confirming the assertions of Bhamare et al. (2016) [17]. From current study, an ionic imbalance could result in increased body temperature and alienation in blood chemistry. Similar findings were observed for afternoon rectal temperature and temperature change at starter phase. An ideal DEB is that which could limit the fluctuations in body or rectal temperature caused by environmental pressure. These results contradict the findings of Borges et al. (2003) [5] who noted that the internal body temperature of heat-stressed broiler chickens decreased linearly as the DEB increased, and that birds fed 240 and $360 \mathrm{mEq} / \mathrm{kg}$ DEB had the lowest temperature and smallest body heat variation from morning to afternoon during summer conditions (Max: $31^{\circ} \mathrm{C}$, Min: $23^{\circ} \mathrm{C}$; $\mathrm{RH}, 75.5 \%$ ). Although, this was attributed to increased water consumption by these birds and presumably heat dissipation and the efficiency in heat evaporative heat loss also increased with increased water intake.

From current study, Na intake was lowest in birds on $210 \mathrm{mEq} / \mathrm{kg}$ and highest in those on $360 \mathrm{mEq} / \mathrm{kg}$ DEB. K intake in birds on $330 \mathrm{mEq} / \mathrm{kg}$ and $360 \mathrm{mEq} / \mathrm{kg}$ was significantly higher compared to other DEB treatments. The mechanism behind this uptake could be linked to the regulatory process of the sodium-potassium pump. The sodium-potassium pump bounds ATP and 3 intracellular $\mathrm{Na}^{+}$ions. The phosphorylation of the pump occurs after the hydrolysis of ATP at a highly conserved aspartate residue to release of ADP. An electric potential results from the lowered permeability of sodium to plasma membrane than it is to potassium ions. The electrical and concentration gradient established by the sodium-potassium ATPase supports not only the cell resting potential but the action potentials of nerves and muscles. Export of $\mathrm{Na}$ from the cell allows for several transporters, which import glucose, amino acids and other nutrients into the cell. An osmotic gradient that drives the absorption of water is created by the translocation of $\mathrm{Na}$ from one side to the other of an epithelium. In the gut, sodium is transported out of the resorbing cell on the blood side via the $\mathrm{Na}^{+}-\mathrm{K}^{+}$pump, whereas, on the resorbing side, the $\mathrm{Na}^{+}$-Glucose symporter uses the created $\mathrm{Na}^{+}$gradient as a source of energy to import both $\mathrm{Na}^{+}$and glucose, which is far more efficient than simple diffusion (Chatterjea and Shinde, 2008) [18]. Absorption of chloride takes place in small intestines. The mechanism of chloride uptake appears to depend on an exchange process with the $\mathrm{HCO}_{3}$, and is important in the production of hydrogen chloride in the gastric juice and in chloride shift during respiration (Harper, 2003) [19]. 


\section{Conclusion}

European production index of starter chicks could be enhanced by increasing the level of $\mathrm{K}$ intake through dietary supplementation up to $1.02 \%$. Weight gain is adversely affected by sharp changes in temperature as a negative correlation existed between these parameters at finisher phase. Increase in water intake may not be a remedial tool in lowering sharp fluctuations in body temperature as no significant correlation existed between these parameters. However, sharp fluctuation in body temperature of broilers was reduced with DEB of 330, 270 and 240 $\mathrm{mEq} / \mathrm{kg}$ at prestarter, starter and finisher phases, respectively.

\section{Acknowledgements}

The profound moral and financial supports of Engr. and Mrs Popoola are hereby acknowledged.

\section{Conflicts of Interest}

The authors declare no conflicts of interest regarding the publication of this paper.

\section{References}

[1] De Rensis, F. and Scaramuzzi, R.J. (2003) Heat Stress and Seasonal Effects on Reproduction in the Dairy Cow-A Review. Theriogenology, 60, 1139-1151. https://doi.org/10.1016/S0093-691X(03)00126-2

[2] Perissinotto, M., Moura, D.J. and Cruz, V.F. (2007) Avaliação da produção de leite em bovinos utilizando diferentes sistemas de climatização. Revista de Ciências Agrárias, 30, 135-142.

[3] Daghir, N.J. (2009) Nutritional Strategies to Reduce Heat Stress in Broilers and Broiler Breeders. Lohmann Information, 44, 6-15.

[4] Athira, P., Ratnakaran, V., Sejian, V., Sanjo, J., Shalini, V., Bagath, M., Krishnan, G., Beena, V., Indira Devi, P., Girish, V. and Bhatta, R. (2017) Behavioral Responses to Livestock Adaptation to Heat Stress Challenges. Asian Journal of Animal Sciences, 11, 1-13. https://doi.org/10.3923/ajas.2017.1.13

[5] Borges, S.A., Fischer Da Silva, A.V., Ariki, J., Hooge, D.M. and Cummings, K.R. (2003) Dietary Electrolyte Balance for Broiler Chickens Exposed to Thermoneutral or Heat-Stress Environments. Poultry Science, 82, 428-435. https://doi.org/10.1093/ps/82.3.428

[6] Mongin, P. (1981). Recent Advances in Dietary Cation Anion Balance: Applications in Poultry. Proceedings of the Nutrition Society, 40, 285-294. https://doi.org/10.1079/PNS19810045

[7] Murakami, A.E., Rondon, E.O.O., Martins, E.N., Pereira, M.S. and Scapinello, C. (2001). Sodium and Chloride Requirements of Growing Broiler Chickens (Twenty-One to Forty-Two Days of Age) Fed Cornsoybean Diets. Poultry Science, 80, 289-294. https://doi.org/10.1093/ps/80.3.289

[8] Popoola, I.O. and Iyayi, E.A. (2018) Response of Heat-Stressed Broiler Chicks to Varying Dietary Electrolyte Balance at Prestarter and Starter Phases. Proceeding of 43 rd Annual Conference of the Nigerian Society for Animal Production, Owerri, 18-22 March 2018, 189-191. 
[9] Popoola, I.O., Popoola, O.R., Ojeniyi, M.O., Olajide, O.O. and Iyayi, E.A. (2020) The Roles of Key Electrolytes in Balancing Blood Acid-Base and Nutrient in Broiler Chickens Reared under Tropical Conditions. Natural Science, 12, 4-11. https://doi.org/10.4236/ns.2020.121002

[10] Popoola, I.O., Oshibanjo, D.O., Popoola, O.R., Okuneye, T.A., Ilaboya, I.I. and Iyayi, E.A. (2019) Effect of Dietary Electrolyte Balance on Water Intake, Litter Moisture and Production of Broiler Chicks at Prestarter and Starter Phases. Open Journal of Animal Sciences, 9, 472-480. https://doi.org/10.4236/ojas.2019.94036

[11] Lacroix, R.L., Keeney, D.R. and Welsh, L.M. (1970) Potentiometric Titration of Chloride in Plant Tissue Extracts Using the Chloride Ion Electrode. Communications in Soil Science and Plant Analysis, 1, 1-6. https://doi.org/10.1080/00103627009366233

[12] Statistical Analysis System (2012) SAS Users Guide: Statistics. SAS Institute Inc., Cary.

[13] Yalçin, S., Özkan, S., Türkmut, L. and Siegel, P.B. (2001) Responses to Heat Stress in Commercial and Local Broiler Stocks. 1. Performance Traits. British Poultry Science, 42, 149-152. https://doi.org/10.1080/00071660120048375

[14] Deep, N. and Cahaner, A. (2001) Genotype-by-Environment Interaction with Broiler Genotypes Differing in Growth Rate: 2. The Effects of High Ambient Temperature on Dwarf Versus Normal Broilers. Poultry Science, 80, 541-548. https://doi.org/10.1093/ps/80.5.541

[15] Plavnik, I. and Yahav, S. (1998) Effect of Environmental Temperature on Broiler Chickens Subjected to Growth Restriction at an Early Age. Poultry Science, 77, 870-872. https://doi.org/10.1093/ps/77.6.870

[16] Sejian, V., Maurya, V.P. and Naqvi, S.M.K. (2012) Effect of Walking Stress on Growth, Physiological Adaptability and Endocrine Responses in Malpura Ewes in a Semi-Arid Tropical Environment. International Journal of Biometeorology, 56, 243-252. https://doi.org/10.1007/s00484-011-0420-y

[17] Bhamare, K.S., Dildeep, V., Senthil, M.S. and Chavan, S.J. (2016) Nutritive Evaluation of Cashew Apple Waste in Broilers. International Journal of Science and Nature, 7, 629-632.

[18] Chatterjea, M.N. and Shinde, R. (2008) Textbook of Medical Biochemistry. 7th Edition, Jaypee Brothers Medical Publishers, New Delhi.

[19] Harper, H.A. (2003) Harper's Illustrated Biochemistry. A Lange Medical Book, International, 26th Edition. 